\title{
Ultrastructural changes during the symbiotic seed germination of Gastrodia elata with fungi, with emphasis on the fungal colonization region
}

Yuan-Yuan Li ${ }^{1,2}$, Shun-Xing Guo ${ }^{2^{*}}$ and Yung-I Lee $3,4^{*}$ (D)

\begin{abstract}
Background: Gastrodia elata is a fully mycoheterotrophic orchid and has long been used in traditional Chinese medicine. The life cycle of $G$. elata requires an association with two different fungi-Mycena for seed germination and Armillaria for tuber growth. The association with Armillaria is representative of the phytophagous type of orchid mycorrhiza: the intracellular hyphae are lysed without forming condensed pelotons. However, whether the association with Mycena during seed germination belongs to the same type of orchid mycorrhiza is unknown.

Results: Histological and ultrastructural studies revealed several notable features in different developmental stages. First, a thickened cell wall with papillae-like structures appeared during fungal penetration in the suspensor end cell, epidermal cells and cortical cells of germinating embryos. In addition, the formation of two distinctive cell types in the colonized region of a protocorm (i.e., the passage canal cell filled with actively growing fungal hyphae) can be observed in the epidermal cell, and the distinctive digestion cell with a dense cytoplasm appears in the cortex. Finally, within the digestion cell, numerous electron-dense tubules form a radial system and attach to degrading fungal hyphae. The fungal hyphae appear to be digested through endocytosis.

Conclusions: The present study provides important structural evidence for the phytophagous type of orchid mycorrhiza in the symbiotic germination of G. elata with Mycena. This case demonstrates a particular nutrient transfer network between G. elata and its litter-decaying fungal partner.
\end{abstract}

Keywords: Mycorrhiza, Mycoheterotrophic orchids, Phytophagy, Symbiotic germination

\section{Background}

Orchid seeds are minute, and most contain an undifferentiated embryo that lacks a well-defined endosperm (Arditti 1992). The few-celled embryo has small amounts of proteins and lipids and very little sugar (Harrison

\footnotetext{
*Correspondence: sxguo1986@163.com; leeyungi@hotmail.com ${ }^{2}$ Institute of Medicinal Plant Development, Chinese Academy of Medical Sciences \& Peking Union Medical College, Beijing 100193, People's Republic of China

3 Biology Department, National Museum of Natural Science, 40453 Taichung, Taiwan

Full list of author information is available at the end of the article
}

1977; Arditti and Ghani 2000). Because of the lack of nutritional reserves, seed germination in nature completely depends on mycorrhizal fungi, which are believed to provide nutrients required for seed germination and protocorm development (Arditti 1967; Dearnaley 2007). There has been extensive research on symbiotic germination (Rasmussen 1995; Smith and Read 2008).

Previous studies of symbiotic germination have provided considerable information about the interaction between mycorrhizal fungi and orchid seeds, especially structural and ultrastructural changes (Burgeff 1909; Clements 1988; Peterson and Currah 1990; Uetake 
et al. 1992, 1997; Peterson et al. 1998; Chen et al. 2014). As the mycorrhizal fungi penetrate the embryo, they form hyphal coils, known as pelotons, enveloped by the plasma membrane in the host cell. Then, the pelotons collapse and undergo lyses, and the digested products are absorbed by the host cell. These studies focused mainly on green orchids, with a few studies on achlorophyllous orchids.

Gastrodia elata, an orchid used in traditional Chinese medicine, is widely distributed in many Asian countries, including China, Korea and Japan. The dry tuber of $G$. elata (known as Tianma in Chinese) has been used to treat many human illnesses, such as headache, vertigo, hemiplegia and infantile convulsions (Xu and Guo 2000) and has strong potential for treating Alzheimer's and Parkinson's disease (Manavalan et al. 2012). G. elata is a fully mycoheterotrophic orchid species. It sets up a symbiotic relationship with two different compatible mycorrhizal fungi during its life history. For seed germination, Mycena is recruited for stimulating germination and the early stages of protocorm development, then further development of tubers requires Armillaria (Xu and Guo 2000).

Previous studies identified two different histological types of orchid mycorrhiza: tolypophagy, occurring in most orchid species, and phytophagy, found in only a few mycoheterotrophic orchid species (Burgeff 1936; Rasmussen 2002). In G. elata, the association with Armillaria is representative of ptyophagic infection. In the inner cortex cells of G. elata tubers, intracellular hyphae of Armillaria are lysed without forming distinct hyphal coils (Wang et al. 1997). However, for symbiotic seed germination, the fungal partner Mycena forms distinct intracellular hyphal coils in the inner cortex cells of the protocorm, which suggests a tolypophagic relation (Fan et al. 1999).

Despite a reported association between G. elata and Mycena during symbiotic seed germination (Guo and Xu 1990; Xu and Fan 2001; Fan et al. 1999, 2001, 2002), information on serial structural changes in symbiotic seed germination with a defined time scale is still lacking. In this study, based on a defined time frame, we describe the histological and ultrastructural changes during symbiotic seed germination of G. elata. Better understanding the structural development in symbiotic seed germination can provide insights into the interaction between a mycoheterotrophic orchid and mycorrhizal fungi.

\section{Methods}

\section{Plant materials}

The tubers of G. elata with dormant flowers buds were cultivated in a greenhouse at the Institute of Medicinal Plant Development, Chinese Academy of Medical
Sciences \& Peking Union Medical College, Beijing. During May, at anthesis, flowers were pollinated by hand with pollen donated from separate individuals. At 20 days after pollination, capsules prior to dehiscence were collected for symbiotic culture.

\section{Mycorrhizal fungus preparation}

The Mycena strain with good stimulating effect on symbiotic germination of G. elata deposited at the Institute of Medicinal Plant Development was used in this study (Guo and Xu 1990). The Mycena strain was first incubated on potato dextrose agar medium (PDA: potato $200 \mathrm{~g} \mathrm{~L}^{-1}$, glucose $20 \mathrm{~g} \mathrm{~L}^{-1}$, agar $12 \mathrm{~g} \mathrm{~L}^{-1}$, pH 5.2 before autoclaving) in darkness at $25 \pm 1{ }^{\circ} \mathrm{C}$ for 7 days, then actively growing mycelium from the colony margin was used as the fungal inoculum. For the symbiotic germination of G. elata, the mycelia culture medium was prepared by mixing fallen leaves of Quercus with wheat bran at a ratio of $8: 2(\mathrm{v} / \mathrm{v})$, then inoculated with the Mycena inoculum and placed in darkness at $25 \pm 1{ }^{\circ} \mathrm{C}$ until leaves were fully colonized with fungal hyphae.

\section{Symbiotic seed germination}

In a laminar flow hood, Quercus leaves fully colonized with fungal hyphae were first placed on water agar medium in a 9-cm diameter Petri dish. Then capsules were surface sterilized in a $1 \% \mathrm{NaClO}$ solution for $20 \mathrm{~min}$ and cut open to remove seeds for inoculation. Approximately 100 seeds of G. elata removed from capsules were inoculated on the surface of each leaf, then dishes were sealed with paraffin and placed in darkness at $25 \pm 1{ }^{\circ} \mathrm{C}$. Seed germination and the formation of protocorms at each developmental stage were observed and recorded under a stereomicroscope weekly for 12 weeks. Germination was defined as emergence of the embryo from the seed coat (i.e., stage 2 as described by Stewart et al. 2003).

\section{Light microscopy}

The seeds and developing mycorrhizal protocorms were fixed in a solution of $2.5 \%$ glutaraldehyde in $0.1 \mathrm{M}$ phosphate buffer ( $\mathrm{pH}$ 6.8) overnight at room temperature, washed in phosphate buffer three times and dehydrated with an ethanol series, then embedded in Technovit 7100 resin (Kulzer and Co., Germany) as described (Yeung and Chan 2015). Sections of $3 \mu \mathrm{m}$ were cut by use of glass knives of the Reichert-Jung 2040 Autocut rotary microtome. For histological observations, sections were stained with Periodic acid-Schiff (PAS) reaction for total insoluble carbohydrates and counterstained with $0.05 \%$ (w/v) toluidine blue O (TBO) (Yeung 1984). Sections were observed and images were captured by using a CCD camera attached to a light microscope (Axio ImagerA1, Carl Zeiss AG). 


\section{Transmission electron microscopy (TEM)}

Symbiotic protocorms were collected and fixed by highpressure freezing in a high-pressure freezer (Leica EM PACT2) as described ( $\mathrm{Li}$ et al. 2018). The fixed protocorms were exposed to freeze substitution medium (ethanol containing $1 \%$ osmium tetroxide, $0.2 \%$ glutaraldehyde and $0.1 \%$ uranyl acetate) in a Leica Automatic Freeze-Substitution System, then embedded in London Resin White methacrylate resin (London Co., Basingstoke, UK). Ultrathin sections $(70-90 \mathrm{~nm})$ were cut by use of the diamond knife of the Leica Reichert Ultracut $\mathrm{S}$ system (Leica Microsystems GmbH1) and placed on formvar-coated nickel grids for observation under a Philip CM 100 transmission electron microscope (FEI Company2) at $80 \mathrm{kV}$.

\section{Results}

Mature seeds of G. elata had an oval-shaped embryo surrounded by a thin seed-coat layer (Fig. 1a, b). During the first week of inoculation, the embryo slightly enlarged and the embryo cells became highly vacuolated (Fig. 1c, d). By week 2 after inoculation, the embryo continued to enlarge, then ruptured the seed coat to form a protocorm (Fig. 1e, f). At this stage, the cells divided frequently at the apical part of protocorm to generate a meristematic zone, but cells at the basal part of protocorm did not further divide and were colonized by fungal hyphae. After 12 weeks of inoculation, the protocorm elongated further (Fig. 1g), and the fungal colonization was restricted to the basal protocorm (Fig. 1h).

In the early stage of inoculation, fungal hyphae primarily penetrated the suspensor end of an embryo (Fig. 2a). The cell wall of the suspensor end became thickened substantially with the formation of papillae-like structures (Fig. 2b). Within the suspensor end cell, many fungal hyphae remained vigorous, but some were digested (Fig. 2b, c). In the uncolonized embryo cell, the protein bodies were degrading and amyloplasts began to appear (Fig. 2d).

After seed germination, the embryo further enlarged, which resulted in the formation of an ovoid protocorm (Fig. 1e, g). In the basal region of the protocorm, the fungal hyphae coiled to form a loose peloton in epidermal cells, which was absent in cortical cells (Fig. 3a). In epidermal cells, most fungal hyphae remained active, and a few hyphae were digested (Fig. 3b). Penetration of fungal hyphae between the wall of epidermal cells and cortical cells was frequent (Fig. 3c). The fungal wall became

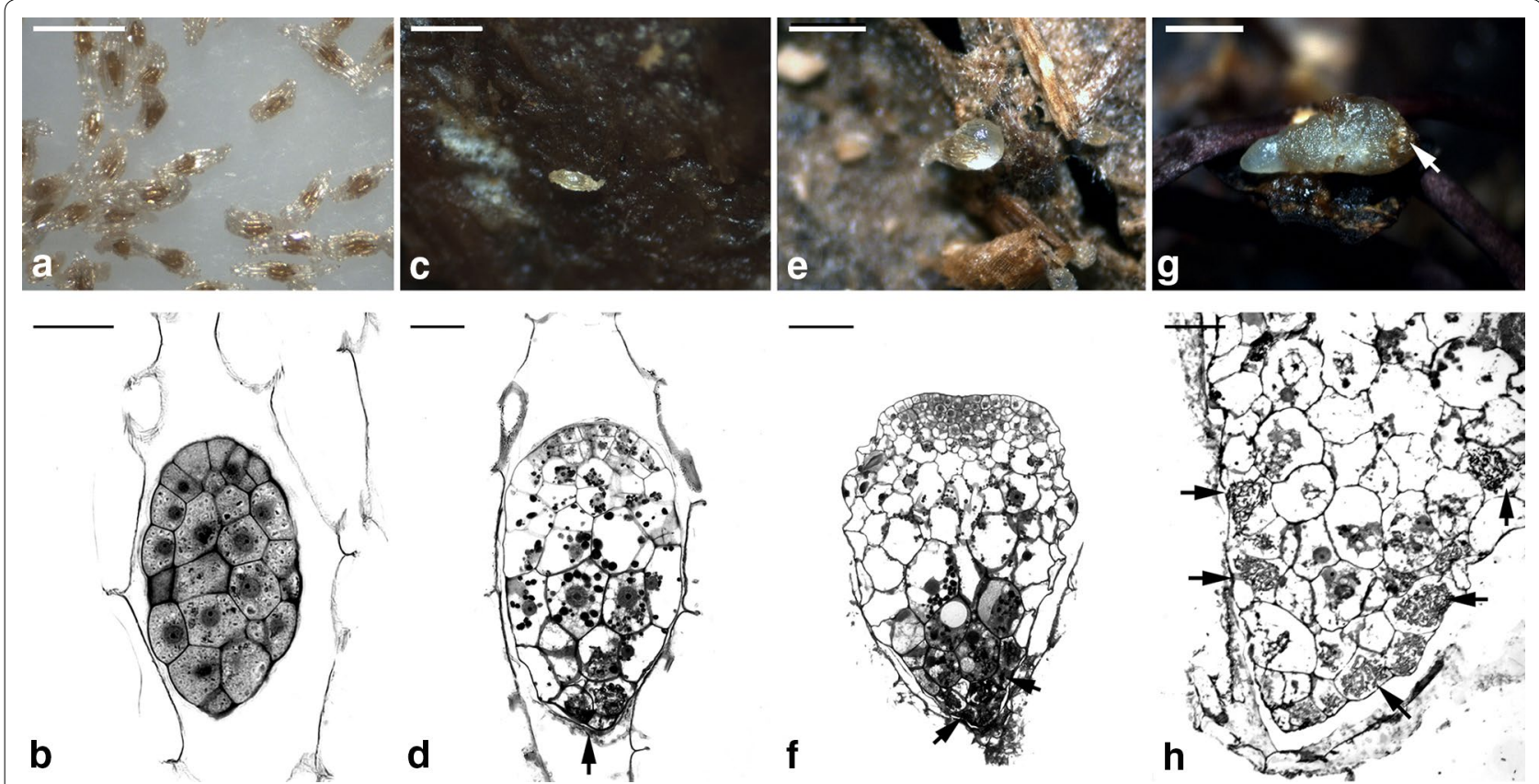

Fig. 1 The symbiotic seed germination of G. elata associated with Mycena. a Mature seeds of G. elata. Scale bar $=0.5 \mathrm{~mm}$. $\mathbf{b}$ Light micrograph of mature seed. The embryo is covered by a thin testa. Scale bar $=50 \mu \mathrm{m}$. c After 1 week of inoculation, a seed becomes swollen. Scale bar $=0.5 \mathrm{~mm}$. d In the enlarged embryo, fungal hyphae have penetrated the embryo through the suspensor end cell (arrow). Scale bar $=50$ um. e After 2 weeks of inoculation, the embryo has ruptured the seed coat, resulting in the formation of a protocorm. Scale bar $=0.5 \mathrm{~mm}$. $\mathbf{f}$ Fungal hyphae (arrows) have colonized the basal region of the developing protocorm. Scale bar $=100 \mu \mathrm{m}$. $\mathbf{g}$ After 12 weeks of inoculation, the elongated protocorm is observed. The basal region of the protocorm is indicated by an arrow. Scale bar $=1 \mathrm{~mm}$. $\mathbf{h}$ Light micrograph showing the basal region of the elongated protocorm, and fungal hyphae (arrows) are restricted at the basal region. Scale bar $=60 \mu \mathrm{m}$ 


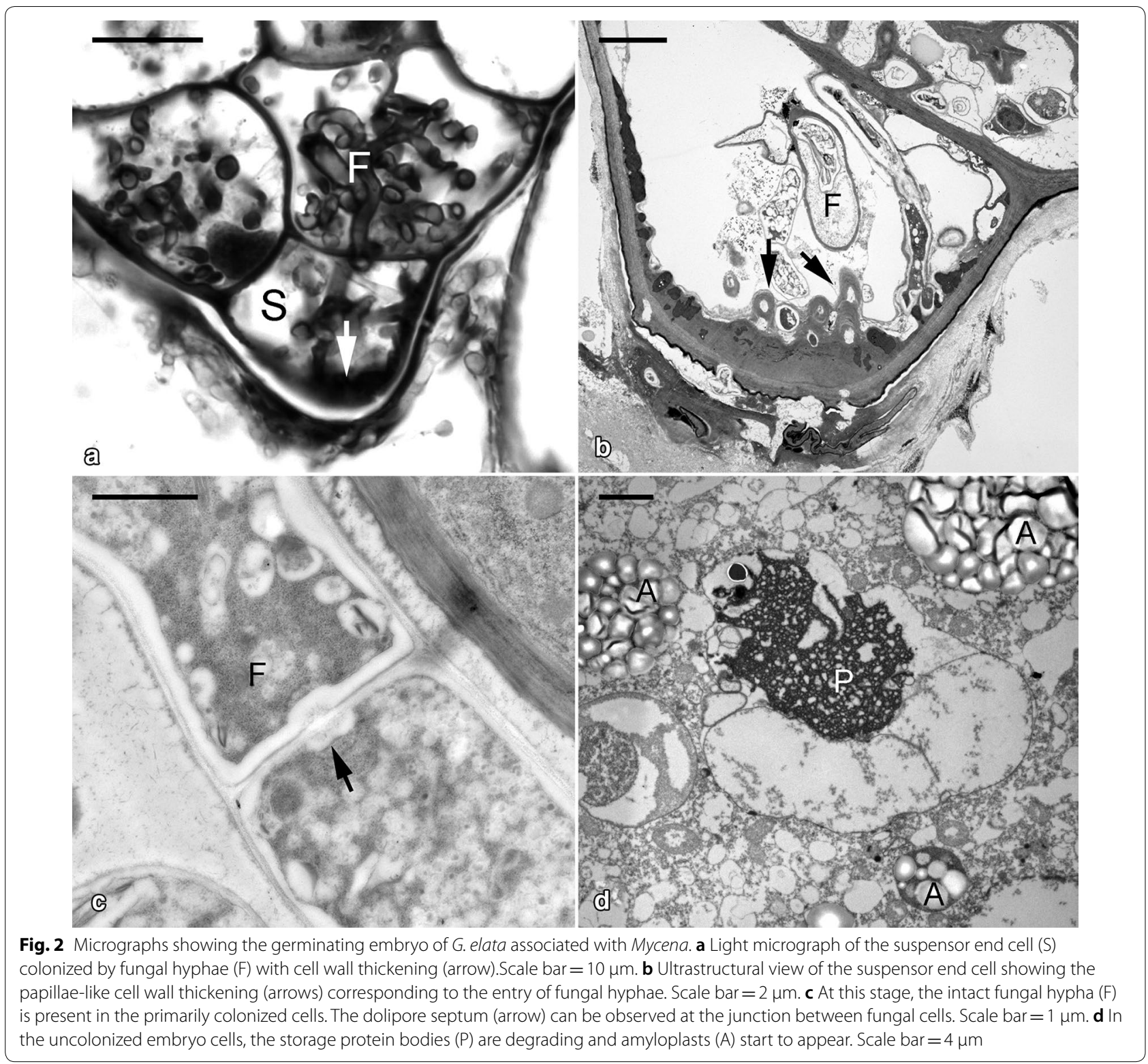

(See figure on next page.)

Fig. 3 Micrographs showing the developing protocorm of G. elata associated with Mycena. a Light micrograph showing the colonized region of a developing protocorm. The epidermal cell (E) contains intact fungal hyphae (arrow), and digested fungal hyphae (arrowhead) are present in the cortical cell (C). Scale bar $=10 \mu \mathrm{m}$. $\mathbf{b}$ In the epidermal cell, a number of intact fungal hyphae (F) are present and are separated from the host cytoplasm by an enveloping interfacial matrix and host plasma membrane. Scale bar $=2 \mu \mathrm{m}$. $\mathbf{c}$ The penetration of fungal hyphae into the cortical cell (arrow). Inside the cortical cell, fungal hyphae are digested and become compressed (arrowheads). Scale bar $=2 \mu \mathrm{m}$. $\mathbf{d}$ After fungal hyphae penetrate the cortical cell, the fungal wall becomes thickened (arrow) by wrapping around additional material of the interfacial matrix and/or host plasma membrane cover. Scale bar $=1 \mu \mathrm{m}$. e In the cortical cell, several electron-dense endocytic tubules (arrows) attach a digesting fungal hypha. In cross sections, the tubular networks appear as numerous vesicles. Scale bar $=1 \mu \mathrm{m}$. $\mathbf{f} \mathrm{A}$ compressed fungal hypha is surrounded by numerous electron-dense endocytic tubules. Scale bar $=1 \mu \mathrm{m}$ 

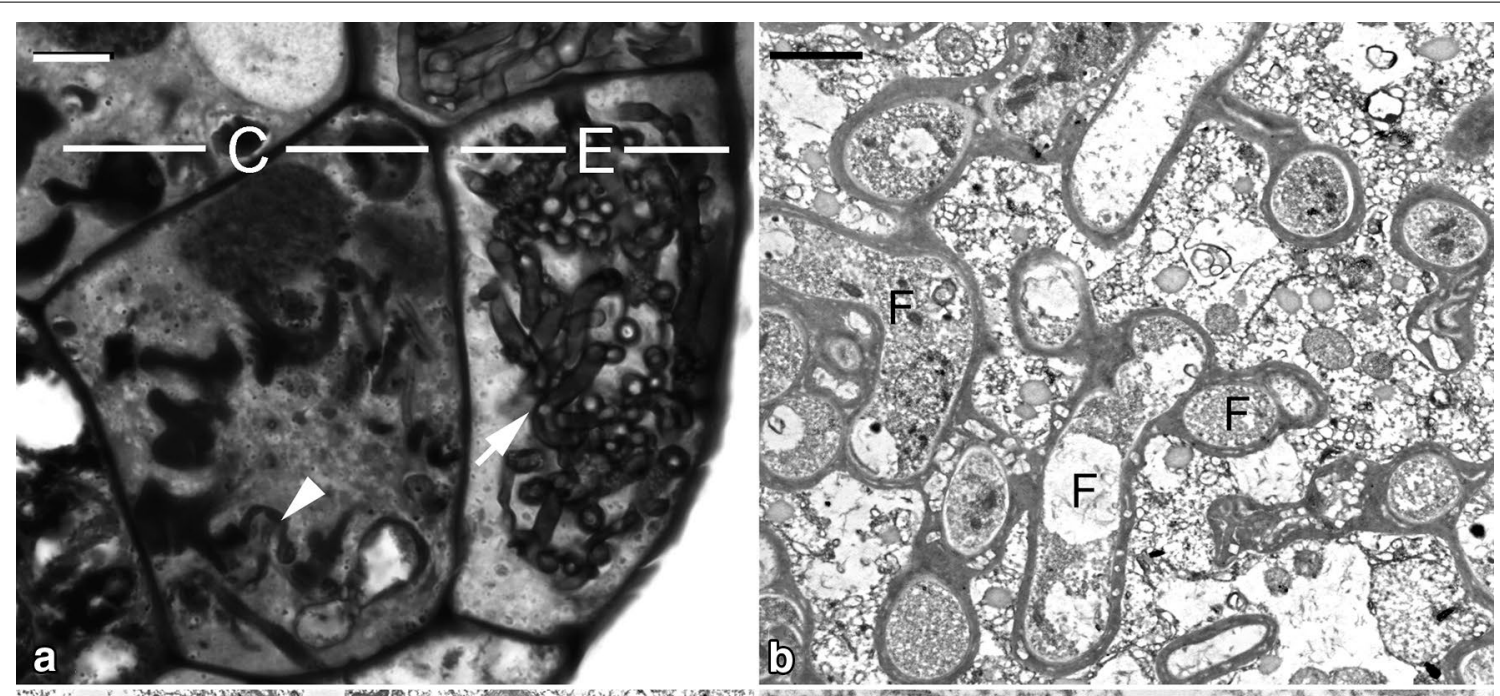

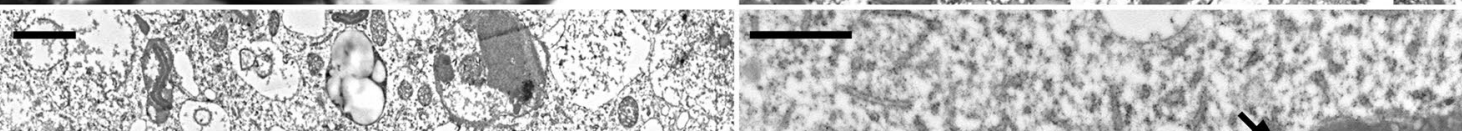
3.

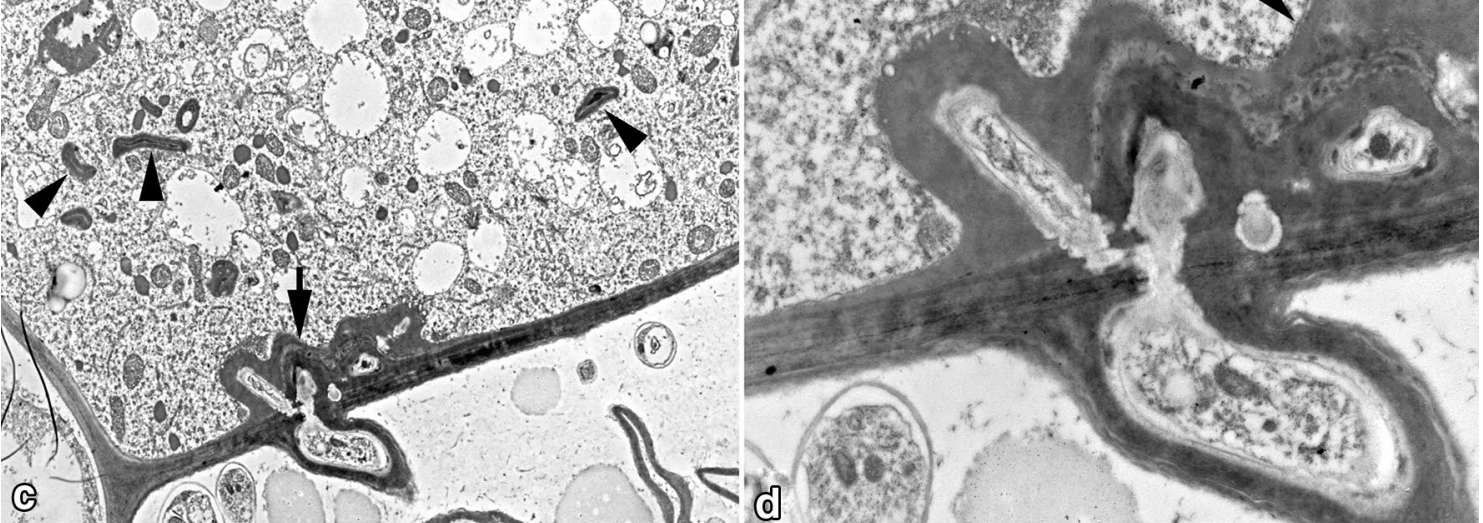

c) 1090

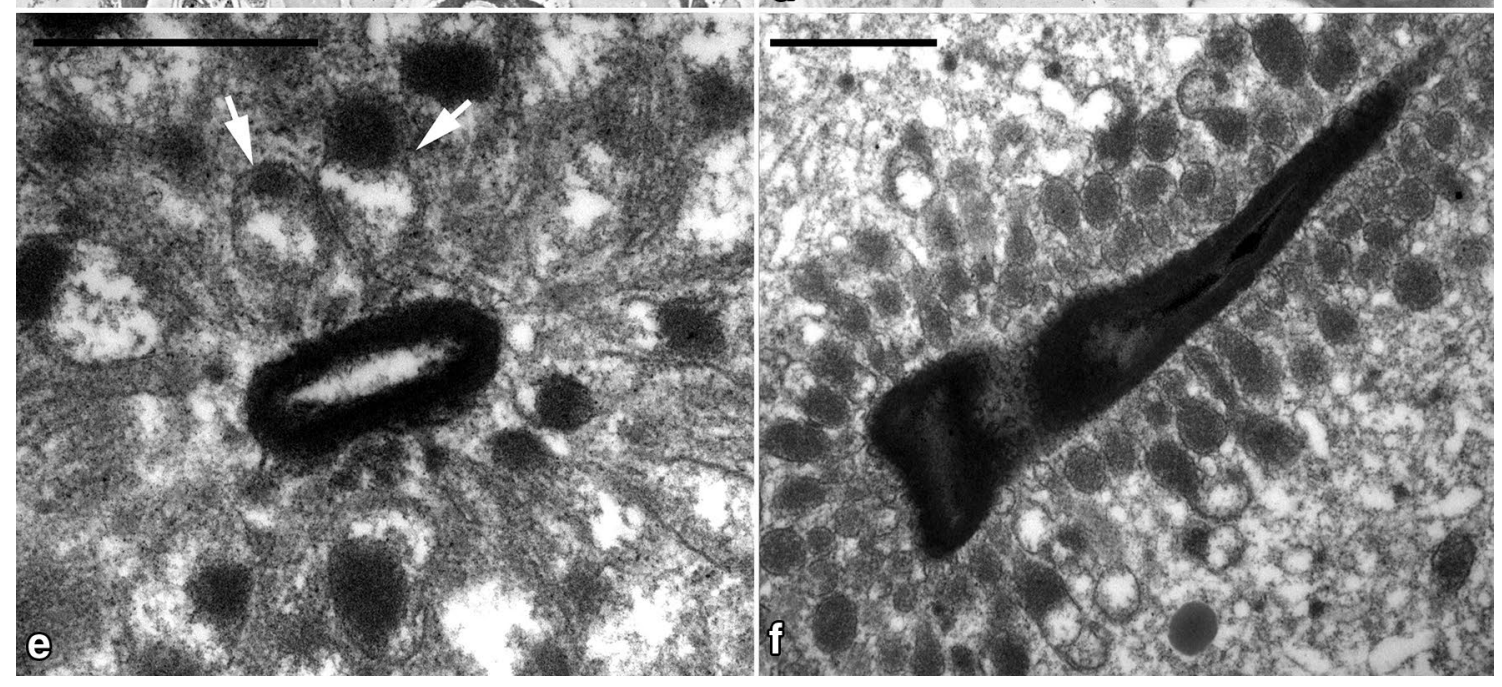


thickened during penetration (Fig. 3d). After entry into the cortical cells, fungal hyphae were soon digested and became compressed. Ultrastructural observations indicated that numerous electron-dense tubular networks appeared and were associated with the surface wall of fungal hyphae (Fig. 3e, f). In cross sections, the tubular networks appeared as vesicles surrounding fungal hyphae (Fig. 3e).

As the protocorm elongated further (Fig. 1h), the fungal hyphae became weak in the epidermal cells at the basal region of a protocorm (Fig. 4a). The cytoplasmic components of these fungal hyphae had broken down and the fungal wall became thickened in the epidermal cells (Fig. 4b). In cortical cells, the volume of vacuoles became larger, and a few compressed fragments of fungal hyphae were visible (Fig. 4a). At this stage, the compressed fungal hyphae assumed a dense appearance and were surrounded by rough endoplasmic reticulum (Fig. 4c). Subsequently, a number of clusters of vesicles appeared and were associated with the fragmented fungal hyphae (Fig. 4d).

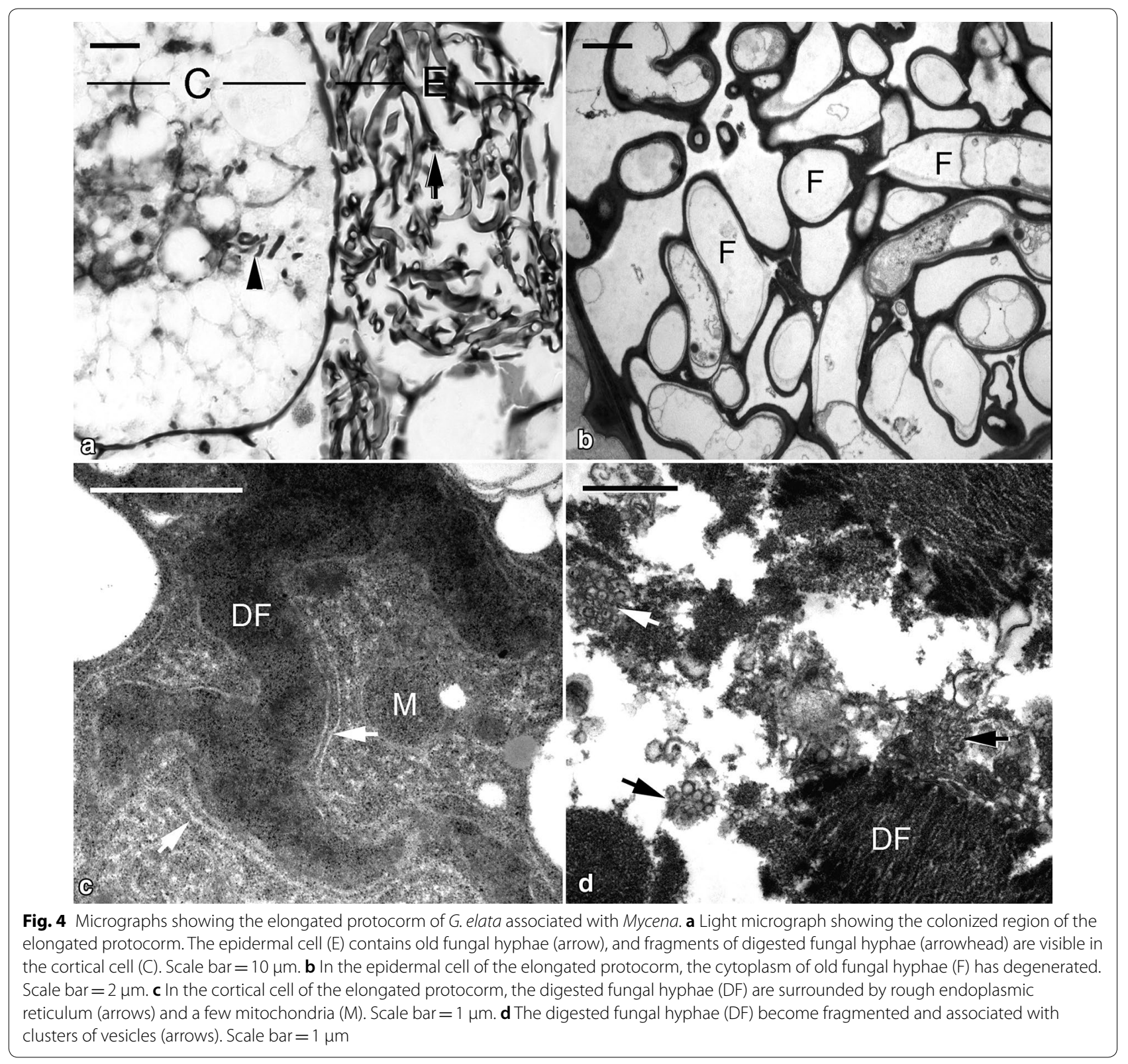




\section{Discussion}

In orchid mycorrhiza, fungal hyphae branch and coil, forming the structure called a peloton within parenchyma cells. Nutrients such as carbon and nitrogen are transferred between orchids and their fungal partners across the interfacial matrix, an apoplastic space produced between the fungal peloton and the orchid cell membrane (Kuga et al. 2014; Fochi et al. 2017). As the peloton is digested, orchids receive fungal nutrients from rupturing fungal hyphae. In our previous studies and the present investigation, the intracellular hyphae of Mycena in cortical cells are digested without forming peloton clumps (Fan et al. 1999; Fig. 3c). These observations suggest a mycorrhizal association between G. elata and Mycena belonging to the phytophagous type of orchid mycorrhiza.

The phytophagous type has been documented in only a few fully mycoheterotrophic orchids, in which fungal hyphae are lysed without forming peloton clumps, then release their cellular contents for use by the orchid (Burgeff 1936; Rasmussen 2002). However, in previous observations, the endocytic vesicles are absent around the lysed fungal hyphae in the symbiotic germination with Mycena (Fan et al. 1999, 2001). One of the notable findings in this study is the presence of tubular endocytic networks attached to the lysed fungal hyphae in the cortical cells of symbiotic protocorms (Fig. 3e, f). During the symbiotic germination (e.g., stages 2 and 3), the fungal hyphae of Mycena from dermal cells penetrate cortical cells and are surrounded by tubular endocytic networks. This structural feature is identical to the phytophagous type of orchid mycorrhiza association characterized in the association of G. elata with Armillaria (Wang et al. 1997; Rasmussen 2002). After the symbiotic germination with Mycena, the growth tube of G. elata requires a switch to a mycorrhizal association with Armillaria. In the inner cortical cells of the growth tube, Armillaria hyphae appear to be lysed as the phytophagous type of orchid mycorrhiza. Ultrastructural studies indicate that as hyphae enter an enlarged digestion cell, the plant plasmalemma and fungal wall (the interfacial matrix) are surrounded by the radiating endocytic tubules to mark the final stage of fungal hyphae breakdown (Wang et al. 1997). Staining with acid phosphatase also suggested the digestive role of these electron-dense tubular networks in cortical cells of G. elata tubers (Wang and Xu 1993).

The lack of endocytic tubules in our previous studies of the symbiotic germination with Mycena is probably due to the degraded tubular networks during sample fixation. In the present study, the mycorrhizal protocorms were prepared by a high-pressure freezing technique, and the dynamic organelle structures were well preserved. A recent transcriptome analysis of G. elata in symbiotic germination with Mycena revealed high up-regulation of a number of genes involved in endocytosis (Zeng et al. 2017). The observations in this study provide the supportive ultrastructural data and suggest nutrient transfer via endocytosis in the phytophagous type of orchid mycorrhiza.

Although the mycorrhizal protocorm of G. elata is small and simple, histology revealed two distinct cell types in the colonization region: a epidermal cell with a degraded cytoplasm and nucleus and filled with actively growing fungal hyphae and a cortical cell with a dense cytoplasm containing lysed fungal hyphae (Fig. 3a). The epidermal cell of the mycorrhizal protocorm is similar to the passage canal cell in the outer cortex of the mycorrhizal tuber in the association with Armillaria, and the cortical cell of the mycorrhizal protocorm resembles the enlarged digestion cell in the inner cortex of the mycorrhizal tuber (Wang et al. 1997). In Gastrodia, the differentiation of the two cell types in mycorrhizal tissue may be an adaptation to these litter- and wood-decaying fungal partners.

Papillae-like cell wall thickening was apparent in walls of epidermal cells of the mycorrhizal protocorm (Fig. 2b) and passage canal cells of the mycorrhizal tuber (Jonsson and Nylund 1979). These cell wall thickenings also mark the penetration sites of fungal hyphae, especially in the suspensor end cell and the adjoining walls between the epidermal and cortical cells (Figs. 2b, 3c). Similar papillae-like cell wall thickening was found in mycorrhizal roots of other Gastrodia species (Martos et al. 2009; Lee et al. 2015). The cell wall thickening could be a defense response to the fungal invasion (Voigt 2014). Moreover, these cells with papillae-like cell wall thickening may be specialized transfer cells in the network of nutrient transport in the mycorrhiza (Pate and Gunning 1972). Further studies of the dynamic changes of cell wall proteins would provide insights into the possible role of the cell wall thickening in mycorrhizal tissue of Gastrodia species.

\section{Conclusions}

The present study provides critical evidence for the phytophagous type of orchid mycorrhiza in symbiotic seed germination of G. elata with Mycena. In symbiotic protocorms, the presence of the passage canal cell in the epidermal cell, the digestion cell in the cortex, and the endocytic tubules for hyphae digestion demonstrate a particular nutrient transfer network in the association with litter-decaying fungal partners.

\section{Acknowledgements}

Authors thank Prof. Jiang-qiang Li of College of Plant Protection/Beijing Key Laboratory of Seed Disease Testing and Control, China Agricultural University, Beijing for reading and commenting on the manuscript. 


\section{Authors' contributions}

LYI and GSX conceived the study. LYI, GSX, LYY and LJQ designed the study. $L Y Y$ and $L Y I$ carried out the experimental work and drafted the manuscript. LJQ, GSX and LYI provided funding. All authors read and approved the final manuscript.

\section{Funding}

This research is supported by the China Postdoctoral Science Foundation (2019M660883) to Yuan-Yuan Li, Natural Science Foundation of China (81573526) to Shun-Xing Guo and by the grant from the CAMS Innovation Fund for Medical Sciences (CIFMS) (2017-I2M-3-013) to Shun-Xing Guo and National Museum of Natural Science, Taiwan, to Yung-I Lee. The funders had no role in the design of the study; collection, analyses, or interpretation of data; writing of the manuscript, or decision to publish.

\section{Availability of data and materials}

Not applicable.

Ethics approval and consent to participate

Not applicable.

\section{Consent for publication}

Not applicable.

\section{Competing interests}

The authors declare that they have no competing interests.

\section{Author details}

${ }^{1}$ College of Plant Protection/Beijing Key Laboratory of Seed Disease Testing and Control, China Agricultural University, Beijing 100193, People's Republic of China. ${ }^{2}$ Institute of Medicinal Plant Development, Chinese Academy of Medical Sciences \& Peking Union Medical College, Beijing 100193, People's Republic of China. ${ }^{3}$ Biology Department, National Museum of Natural Science, 40453 Taichung, Taiwan. ${ }^{4}$ Department of Life Sciences, National Chung Hsing University, 40227 Taichung, Taiwan.

Received: 16 October 2019 Accepted: 30 December 2019 Published online: 12 February 2020

\section{References}

Arditti J (1967) Factors affecting the germination of orchid seeds. Bot Rev 33:1-97 Arditti J (1992) Fundamentals of orchid biology. Wiley, New York

Arditti J, Ghani AKA (2000) Numerical and physical properties of orchid seeds and their biological implications. New Phytol 146:569

Burgeff H (1909) Die Wurzelpiltze der Orchideen, ihreKultur und ihre Leben in der Pflanze. Gustav Fischer, Jena

Burgeff H (1936) Samenkeimung der Orchidéen. Gustav Fischer, Jena

Chen J, Wang H, Liu SS, Li YY, Guo SX (2014) Ultrastructure of symbiotic germination of the orchid Dendrobium officinale with its mycobiont, Sebacina sp. Aust J Bot 62:229-234

Clements MA (1988) Orchid mycorrhizal associations. Lindleyana 3:73-86

Dearnaley JDW (2007) Further advances in orchid mycorrhizal research. Mycorrhiza 17:475-486

Fan L, Guo SX, Xu JT (1999) Interaction between protocorms of Gastrodia elata (Orchidaceae) and Mycena dendrobii in symbiotic germination. Mycosystema 18:219-225

Fan L, Guo SX, Xiao PG (2001) Interaction between Gastrodia elata (Orchidaceae) and Mycena anoectochila during seed germination. Mycosystema 20:539-546

Fan L, Guo SX, Xu JT (2002) Ultrastructural changes during the symbiotic development of Gastrodia elata (Orchidaceae) protocorms associated with Mycena osmundicola. J Cap Norm Univ 23:52-56

Fochi V, Chitarra W, Kohler A, Voyron S, Singan VR, Lindquist EA et al (2017) Fungal and plant gene expression in the Tulasnella calospora-Serapias vomeracea symbiosis provides clues about nitrogen pathways in orchid mycorrhizas. New Phytol 213:365-379

Guo SX, Xu JT (1990) Studies on the cell ultrastructure in the course of Gastrodia elata digesting Mycena osmundicola Lange and Armillaria mellea FR. Acta Mycol Sin 9:218-225
Harrison CR (1977) Ultrastructural and histochemical changes during germination of Cattleya aurantica (Orchidaceae). Bot Gaz 138:41-45

Jonsson L, Nylund JE (1979) Favolaschia dybowskyana (singer) singer (aphyllophorales), a new orchid mycorrhizal fungus from tropical Africa. New Phytol 83:121-128

Kuga Y, Sakamoto N, Yurimoto H (2014) Stable isotope cellular imaging reveals that both live and degenerating fungal pelotons transfer carbon and nitrogen to orchid protocorms. New Phytol 202:594-605

Lee Yl, Yang CK, Gebauer G (2015) The importance of associations with saprotrophic non-rhizoctonia fungi among fully mycoheterotrophic orchids is currently under-estimated: novel evidence from sub-tropical Asia. Ann Bot 116:423-435

Li YY, Chen XM, Zhang Y, Cho YH, Wang AR, Yeung EC, Zeng X, Guo SX, Lee YI (2018) Immunolocalization and changes of hydroxyproline-rich glycoproteins during symbiotic germination of Dendrobium officinale. Front Plant Sci 9:552

Manavalan A, Feng L, Sze SK, Hu JM, Heese K (2012) New insights into the brain protein metabolism of Gastrodia elata-treated rats by quantitative proteomics. J Proteomics 75:2468-2479

Martos F, Dulormne M, Pailler T, Bonfante P, Faccio A, Fournel J, Dubois MP, Selosse MA (2009) Independent recruitment of saprotrophic fungi as mycorrhizal partners by tropical achlorophyllous orchids. New Phytol 184:668-681

Pate JS, Gunning BES (1972) Transfer cells. Ann Rev Plant Physiol 23:173-196

Peterson RL, Currah RS (1990) Synthesis of mycorrhizae between protocorms of Goodyera repens (Orchidaceae) and Ceratobasidium cereale. Can J Bot 68:1117-1125

Peterson RL, Uetake Y, Zelmer C (1998) Fungal symbioses with orchid protocorms. Symbiosis 25:29-55

Rasmussen HN (1995) Terrestrial orchids: from seed to mycotrophic plant. Cambridge University Press, Cambridge

Rasmussen HN (2002) Recent developments in the study of orchid mycorrhiza. Plant Soil 244:149-163

Smith SE, Read DJ (2008) Mycorrhizal symbiosis, 3rd edn. Academic Press, San Diego

Stewart SL, Zettler LW, Minso J, Brown PM (2003) Symbiotic germination and reintroduction of Spiranthes brevilabris Lindley, an endangered orchid native to Florida. Selbyana 24:64-70

Uetake Y, Kobayashi K, Ogoshi A (1992) Ultrastructural changes during the symbiotic development of Spiranthes sinensis (Orchidaceae) protocorms associated with binucleate Rhizoctonia anastomosis group C. Mycol Res 96:199-209

Uetake Y, Earquhar ML, Peterson RL (1997) Changes in microtubule arrays in symbiotic orchid protocorms during fungal colonization and senescence. New Phytol 135:701-709

Voigt CA (2014) Callose-mediated resistance to pathogenic intruders in plant defense-related papillae. Front Plant Sci 5:168

Wang H, Xu JT (1993) A cytochemical study of acid phosphatase in the cortical cell of Gastrodia elata infected by Armillaria mellea. Acta Mycol Sin 12:152-157

Wang H, Wang Z, Zhang F, Liu J, He X (1997) A cytological study on the nutrientuptake mechanism of a saprophytic orchid Gastrodia elata. Acta Bot Sin 9:500-504

Xu JT, Fan L (2001) Cytodifferentiation of the seeds (protocorms) and vegetative propagation corms colonized by mycorrhizal fungi. Acta Bot Sin 10:001

Xu JT, Guo SX (2000) Retrospect on the research of the cultivation of Gastrodia elata B1, a rare traditional Chinese medicine. Chin Med J 113:686-692

Yeung EC (1984) Histological and histochemical staining procedures. In: Vasil IK (ed) Cell culture and somatic cell genetics of plants. Academic Press, Orlando, pp 689-697

Yeung EC, Chan CKW (2015) The glycol methacrylate embedding resinsTechnovit 7100 and 8100 . Plant microtechniques and protocols. Springer International Publishing, Berlin, pp 67-82

Zeng X, Li YY, Ling H, Liu SS, Liu MM, Chen J, Guo SX (2017) Transcriptomic analyses reveal clathrin-mediated endocytosis involved in symbiotic seed germination of Gastrodia elata. Bot Stud 58:31

\section{Publisher's Note}

Springer Nature remains neutral with regard to jurisdictional claims in published maps and institutional affiliations. 